\title{
OPÇÃO MINIMAMENTE INVASIVA LATERAL PARA ARTRODESE INTERSOMÁTICA TÓRACO-LOMBAR
}

\author{
MINIMALLY INVASIVE LATERAL OPTION FOR THORACIC-LUMBAR \\ INTERSOMATIC ARTHRODESIS
OPCIÓN MÍNIMAMENTE INVASIVA LATERAL PARA ARTRODESIS INTERSOMÁTICA TORACOLUMBAR

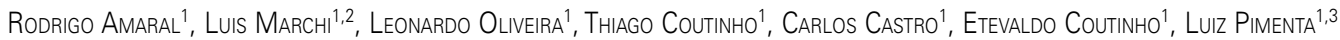

\begin{abstract}
RESUMO
Objetivo: O objetivo deste artigo é mostrar resultados clínicos e radiográficos do acesso lateral transpoas na experiência brasileira em condições degenerativas do disco intervertebral. Métodos: 46 pacientes foram submetidos à fusão intersomática lombar por via lateral. Dentre os casos, 18 eram do sexo masculino e 28 do sexo feminino, com idade média de 57,3 (84-32 anos) e média de IMC de 25,9 \pm 3,1. Todos os pacientes completaram um ano de acompanhamento. Foram coletados exames radiológicos, como raio $\mathrm{X}$ e tomografia computadorizada, exame neurológico e resultados clínicos usando os questionários ODI e VAS (costas e membros inferiores). Resultados: Os procedimentos foram realizados, sem ocorrência de complicações intra-operatórias importantes, em uma média de 103,9 \pm 105,5 minutos e com menos de 50cc de perda sanguínea. Em oito dos 46 procedimentos $(17,4 \%)$ foi utilizada suplementação por parafusos pediculares percutâneos por apresentarem instabilidade segmentar. Foram tratados 80 níveis (de um a cinco níveis) tóraco-lombares (de T12-L1 a L4-L5). Os resultados clínicos avaliados pelos questionários revelaram melhora significante de dor logo após uma semana da cirurgia e da função física após seis semanas. A lordose lombar foi de 36,5 $\pm 14,7$ no pré-operatório para 43,4 $\pm 12,4$ no seguimento de 12 meses. Todos os pacientes apresentaram formação óssea após 12 meses da cirurgia. Sete casos foram revisados (15,2\%), ainda de forma minimamente invasiva devido à estenose persistente (três casos; $6,5 \%$ ), afundamento do espaçador (três casos; $6,5 \%$ ) ou mal-alinhamento de barra da suplementação (um caso; $2,8 \%$ ). Conclusões: Com melhora de parâmetros clínicos e radiológicos, a técnica se mostrou segura e eficaz no tratamento de condições degenerativas da coluna lombar.
\end{abstract}

Descritores: Coluna vertebral, Artrodese; Procedimentos cirúrgicos minimamente invasivos coluna; Coluna lombar; Cirurgia minimamente invasiva.

\begin{abstract}
Objective: The purpose of this article is to show clinical and radiological results of lateral transpsoas approach for degenerative conditions of intervertebral disc in Brazilian practice. Methods: 46 patients have undergone lateral lumbar intersomatic fusion. Eighteen patients were male and 28 female. The mean age was 57.3 years (32 to 84 years), and mean BMI was $25.9 \pm 3.1$. All patients were followed up during one year. Radiology tests, such as X-ray and CT, and neurological tests were performed. The clinical results were obtained using ODI and VAS (back and leg) questionnaires. Results: The procedures were performed during an average time of $103.9 \pm 105.5$ minutes, and less than $50 \mathrm{~mL}$ of blood loss occurred. Also, no significant intraoperative complication occurred. Supplementation with percutaneous pedicle screws due to segmental instability was used in eight of the 46 procedures (17.4\%). Eighty thoracic-lumbar (from T12-L1 to L4-L5) levels (from one to five) were treated. According to the questionnaires, the clinical results showed significant pain and physical function improvement after one week and six weeks of surgery, respectively. The lumbar lordosis changed from $36.5 \pm 14.7$ before surgery to $43.4 \pm 12.4$ during one year follow-up. All patients showed bone formation 12 months after surgery. Seven cases were revised (15.2\%), using minimally invasive approach due to persistent stenosis (three cases, 6.5\%), depressed spacer (three cases, 6.5\%), and malalignment of supplemental rod (one case, $2.8 \%$ ). Conclusions: This technique showed to be a safe and efficacious treatment for degenerative diseases of lumbar spine as demonstrated by the improvement of clinical and radiological parameters.
\end{abstract}

Keywords: Vertebral spine, Arthrodesis; Minimally invasive surgical procedures of spine: Lumbar spine; Minimally invasive techniques.

\section{RESUMEN}

Objetivo: El objetivo de este artículo es mostrar resultados clínicos y radiográficos del acceso lateral transpolar, en la experiencia brasileña, en condiciones degenerativas del disco intervertebral. Métodos: 46 pacientes fueron sometidos a fusión intersomática lumbar por vía lateral. Dentro de los casos, 18 eran del sexo masculino y 28 del sexo femenino, con edad promedio de 57,3 años (84-32) y promedio de IMC de 25,9 \pm 3,1. Todos los pacientes completaron un año de acompañamiento. Fueron realizados exámenes radiológicos, como rayos $X$ y tomografía computadorizada, examen neurológico y se obtuvieron resultados clínicos usando los cuestionarios ODI y VAS (espaldas y miembros inferiores). Resultados: Los procedimientos fueron realizados, sin ocurrencia de importantes complicaciones intraoperatorias, en un promedio de 103,9 \pm 105,5 minutos y con menos de 50 cc de pérdida sanguínea. En ocho de los 46 procedimientos (17,4\%) se utilizó suplementación mediante tornillos pediculares percutáneos porque se presentaba instabilidad de segmento. Se trataron 80 niveles (de uno a cinco niveles) toracolumbares (de T12-L1 a L4-L5). Los resultados clínicos, a los que se tuvo acceso por los cuestionarios, revelaron mejoría significativa del dolor, luego después de una semana de la cirugía y mejor función física después de 12 meses. La lordosis lumbar fue 36,5 \pm 14,7 en el preoperatorio yendo para 43,4 \pm 12,4 durante el seguimiento de 12 meses. Todos los pacientes presentaron formación ósea después de 12 meses de la cirugía. Siete casos tuvieron nueva intervención (15,2\%), aunque de manera mínimamente invasiva, debido a la estenosis persistente (tres casos; 6,5\%), hundimiento del espaciador (tres casos; 6,5\%) o mala alineación de la barra de suplementación (un caso; 2,8\%). Conclusiones: con mejora de parámetros clínicos y radiológicos, la técnica se mostró segura y eficaz en el tratamiento de condiciones degenerativas de la columna lumbar.

Descriptores: Columna vertebral; Artrodesis; Procedimientos quirúrgicos mínimamente invasivos de columna; columna lumbar; artrodesis; cirugía mínimamente invasiva.

1. Instituto de Patologia da Coluna - São Paulo, SP, Brasil.

2. Departamento de Diagnóstico por Imagem - Universidade Federal de São Paulo (UNIFESP) - São Paulo, SP, Brasil.

3. Departamento de Neurocirurgia - UCSD - San Diego - EUA.

Trabalho realizado no Instituto de Patologia de Coluna (IPC) - São Paulo, SP, Brasil

Correspondência: Luiz Pimenta - Rua Vergueiro 1421 sala 305, São Paulo, SP, 04101000, Brasil. E-mail: luizpimenta@luizpimenta.com.br 


\section{INTRODUÇÃO}

A artrodese lombar vem sendo utilizada amplamente para diferentes condições patológicas da coluna consequentes de degeneração, trauma ou neoplasia. Relatos do começo do século passado já descreveram a artrodese lombar, sendo esta por via anterior (ALIF, por Capener em 1930) ${ }^{1}$ ou posterior (PLIF, por Cloward em $1945)^{2}$. Seguindo estes ideais, a fusão intersomática lombar por via transforaminal (TLIF) foi aperfeiçoada e popularizada por Harms e Jeszensky ${ }^{3}$, a qual utiliza abordagem menos invasiva por via unilateral posterior. Desde 1991, quando Obenchain ${ }^{4}$ descreveu a primeira discectomia lombar laparoscópica, o campo da cirurgia minimamente invasiva da coluna continuou a evoluir. Cirurgião e paciente igualmente foram atraídos pelas vantagens da cirurgia minimamente invasiva, incluindo o menor trauma dos tecidos durante a abordagem cirúrgica, menos dor pós-operatória, estadia hospitalar mais curta e o retorno mais rápido às atividades da vida diária ${ }^{5-10}$. E mais recentemente, Ozgur et $\mathrm{al}^{5}$ descreveram o acesso lateral a $90^{\circ}$ à coluna lombar anterior (XLIF) como opção artrodese intersomática.

A fusão intersomática tem mostrado diversas vantagens teóricas sobre a fusão posterolateral tradicional do ponto de vista biomecânico ${ }^{11,12}$. Porém, existem complicações importantes de abordagens posteriores, que podem incluir a pseudartrose, o deslocamento do enxerto, violação neurológica por danos diretos ao saco dural ou raízes nervosas ${ }^{13}$, e a ressecção muscular extensa de abordagens abertas também podem levar à atrofia, disfunção crônica da musculatura paravertebral e síndrome pós-cirúrgica da coluna lombar ${ }^{14-17}$. Um acesso por via anterior permite a restauração do espaço de disco, da lordose lombar e do alinhamento da coluna sem comprometer a bandas de tensão posterior ${ }^{18-22}$, mas as taxas de complicação são da gama de $2,8-80 \%$ e mais frequentemente envolvem lesões vasculares, lesões neurológicas somáticas, trombose venosa profunda e disfunção sexual|23-26. A taxa de complicação aumenta significativamente se a abordagem anterior é utilizada para a cirurgia de revisão ${ }^{27,28}$.

Este trabalho tem como objetivo relatar resultados clínicos e radiográficos do acesso lateral transpoas na experiência brasileira.

\section{MÉTODOS}

Estudo transversal de prontuários médicos e de imagens radiológicas de casos tratados com fusão intersomática através de acesso lateral transpsoas minimamente invasivo. Foram incluídos casos de degeneração do disco intervertebral com estenose e/ou instabilidade, doença do nível adjacente, pseudoartrose pós artrodese póstero-lateral, espondilolistese de baixo grau ou infecção discal. Todos os casos tiveram no mínimo um ano de acompanhamento após a cirurgia. Foram excluídos casos de escoliose, assim como procedimentos com outros acessos concomitantes (ex. ALIF ou descompressão posterior)

Foram incluídos 46 casos, 18 eram do sexo masculino e 28 do sexo feminino, com idade média de 57,3 (84-32 anos) e média de IMC de 25,9 $\pm 3,1$. Ao todo foram tratados 80 níveis (de um a cinco níveis concomitantes) tóraco-lombares (de T12-L1 a L4-L5).

A utilização de suplementação posterior com parafusos pediculares percutâneos foi aplicada nos casos onde existia instabilidade segmentar, observada em radiografia dinâmica lateral. Em oito casos (17.4\%) foi utilizada a suplementação. Os resultados cirúrgico-demográficos estão resumidos na Tabela 1.

O procedimento cirúrgico foi realizado por um acesso retroperitoneal lateral minimamente invasivo ${ }^{5}$, no qual o paciente é posicionado em decúbito lateral a $90^{\circ}$ e a mesa flexionada para aumentar a distância entre a crista ilíaca e a costela. A mesa cirúrgica deve ser radio-opaca para utilização de fluoroscopia intra-operatória. Dilatadores tubulares progressivos descem pelo espaço retroperitoneal até a coluna lombar por entre as fibras do músculo psoas maior. Com constante monitorização eletromiográfica (EMG) (Neurovision JJB NuVasive, RJ, Brasil) para avaliar a proximidade das raízes do plexo lombar um afastador trilaminar (MaXcess, NuVasive, RJ,
Brasil) é aberto e o corredor operatório é aberto. Sob visão direta e tridimensional, é feita discectomia para posterior inserção de implante de peek (poli-éter-éter-cetona) (CoRoent XL, NuVasive, RJ, Brasil) preenchido com exerto ósseo sintético de fosfato de cálcio.

Tabela 1 - Dados demográficos e cirúrgicos.

\begin{tabular}{|c|c|}
\hline Pacientes & 46 \\
\hline Idade (anos) & $57.3 \pm 14.3$ \\
\hline \multicolumn{2}{|l|}{ Gênero } \\
\hline feminino & $28(60,9 \%)$ \\
\hline masculino & $18(29,1 \%)$ \\
\hline IMC $\left(\mathrm{m} / \mathrm{kg}^{2}\right)$ & $25.9 \pm 3.1$ \\
\hline \multicolumn{2}{|l|}{ Indicação primária } \\
\hline DDD & $20(43,5 \%)$ \\
\hline espondilolistese & $14(30,4 \%)$ \\
\hline pseudoartrose & $5(10,9 \%)$ \\
\hline doença do nível adjacente & $5(8,7 \%)$ \\
\hline discite & $2(4,3 \%)$ \\
\hline Níveis tratados & 80 \\
\hline T12L1 & $3(3,8 \%)$ \\
\hline L1L2 & $6(7,5 \%)$ \\
\hline L2L3 & $9(11,3 \%)$ \\
\hline L3L4 & $19(23,8 \%)$ \\
\hline L4L5 & $4353,8 \%)$ \\
\hline Casos com suplementação posterior & $8(17,4 \%)$ \\
\hline Casos sem suplementação & $38(82,6 \%)$ \\
\hline Perda sanguínea (ml) & $<50$ \\
\hline Tempo de cirurgia (min) & $103.9 \pm 105.5$ \\
\hline
\end{tabular}

Os resultados clínicos foram coletados através dos questionários Oswestry Disability Index (ODI) ${ }^{29}$ para medir o nível de dor e incapacidade associadas com diversas atividades diárias, e Escala Visual Analógica (VAS) para avaliar a intensidade da dor para dores nas costas e pernas.

Exames de tomografia computadorizada e radiografias foram usados para avaliar a fusão em 12 meses após a cirurgia. As imagens foram avaliadas por quatro cirurgiões de coluna. A fusão foi definida como ponte de ligação óssea dos corpos vertebrais adjacentes, quer através dos implantes ou ao redor dos implantes, menos de $5^{\circ}$ de movimento angular, menor ou igual a $3 \mathrm{~mm}$, e uma ausência de linhas radiolúcidas.

Os dados foram coletados antes da cirurgia, no intra-operatório e em uma e seis semanas, três, seis e 12 meses após a cirurgia. Complicações e cirurgias de revisão também foram registradas.

Os dados foram analisados usando o teste $t$ de Student. Valores de $p$ inferiores a 0,05 foram considerados estatisticamente significativos.

\section{RESULTADOS}

Os procedimentos foram realizados, sem ocorrência de complicações intra-operatórias importantes, em uma média de 103,9 \pm 105,5 minutos e com menos de 50cc de perda sanguínea. A média de estadia hospitalar foi de 1,3 \pm 0,9 dias. Os dados cirúrgicos estão resumidos na Tabela 1.

Os resultados clínicos acessados pelos questionários mostraram melhora de sintomas clínicos. No grupo estudado, os níveis de dor acessados pelo VAS (Figuras 1A e 1B) foram significativamente aliviados $(p<0,001)$ logo na primeira visita pós-operatória e se mantiveram baixos até o último seguimento. A restrição física mostrada pelo ODI (Figura1C) foi significantemente abaixada a partir da visita de seis semanas $(p<0,001)$ e mantida abaixo deste nível. 


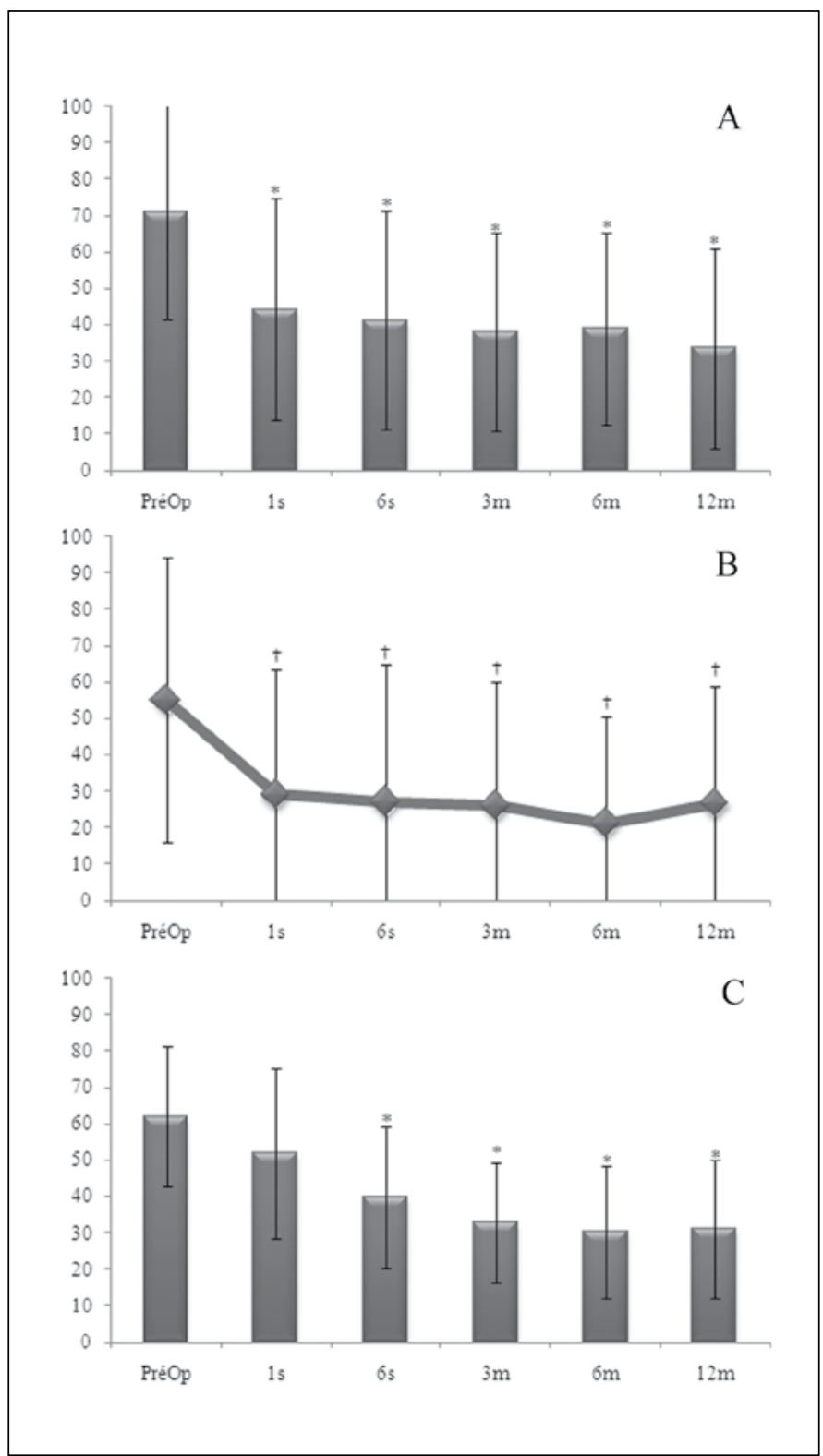

Figura 1. Resultados clínicos acessados pelos questionários clínicos. (A) Resultados de dor nas costas do VAS. * $p<0,001$. (B) Resultados de dor nos membros inferiores do VAS. $\dagger p<0,04$. (C) Resultados de restrição física acessada por ODI. * $p<0,001$.

A média de lordose lombar do grupo estudado era de 36,5 \pm 14,7 no pré-operatório e aumentou com o acesso lateral $(p<0,015)$, sendo que no seguimento de 12 meses para 43,4 \pm 12,4 (Figura 2).

Além da acentuação da curvatura lombar, nos casos de espondilolistese, foi observada redução $(p<0,001)$ do escorregamento vertebral de $18,3 \pm 7,9 \%$ no pré-operatório para $7,0 \% \pm$ 4,2\% (Figura 3).

A avaliação de exames de tomografia computadorizada e de raios-X de 12 meses de acompanhamento revelou que 93,5\% dos níveis tratados apresentaram formação óssea após 12 meses da cirurgia. Um exemplo de caso evidenciando a fusão resultante é mostrado na Figura 4.

Sete casos (15.2\%) foram revisados (Tabela 2). As revisões devido à estenose persistente (três casos; 6.5\%) e afundamento do espaçador (três casos; 6.5\%) foram realizadas com um mini-open para uma descompressão over-the-top e suplementação com parafusos percutâneos. Um caso (2,8\%) evoluiu com mau alinhamento de barra da suplementação e foi reacessado para correção da curvatura da barra.

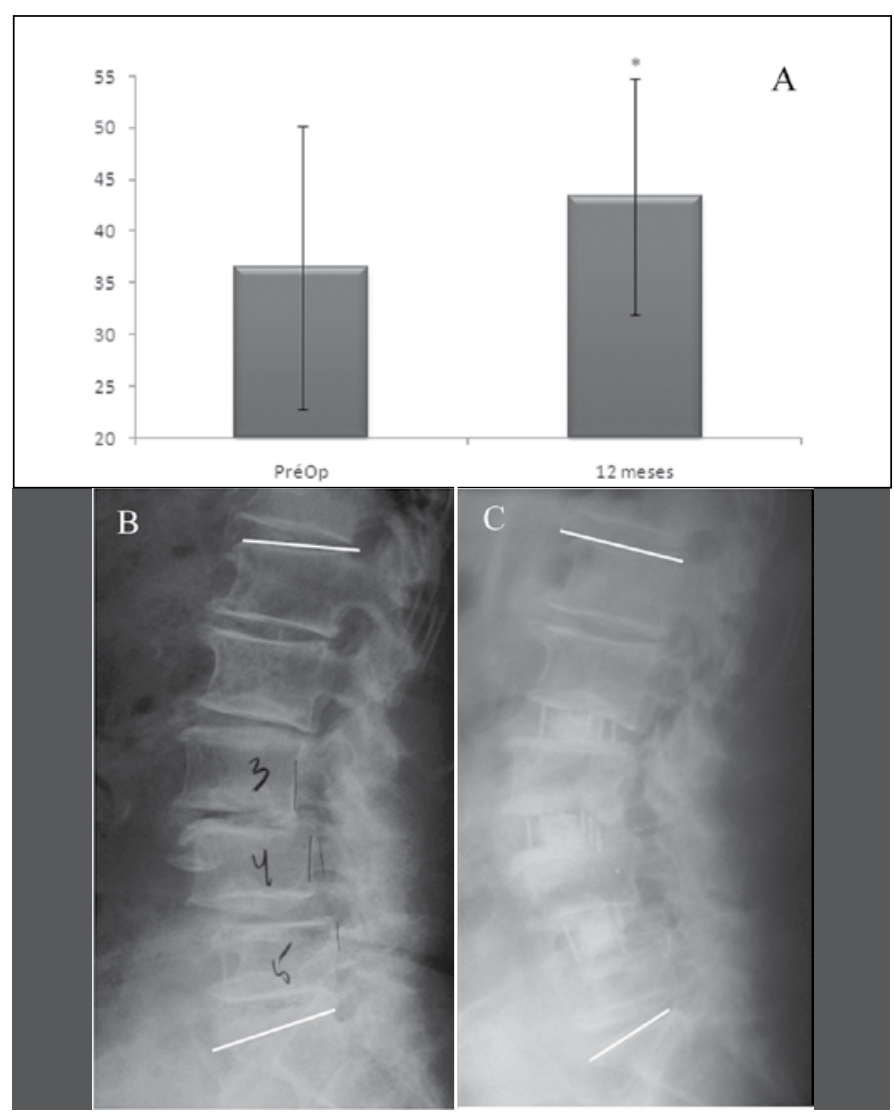

Figura 2. Aumento da lordose lombar após a cirurgia. (A) São mostrados valores médios em graus da lordose lombar da avaliação pré-operatória e de 12 meses após cirúrgia. * $p<0,015$. (B) Imagem de raios-x pré-operatório e (C) de 12 meses mostrando exemplo de caso com aumento da lordose lombar após fusão intersomática trando exemplo de caso
em três níveis lombares.
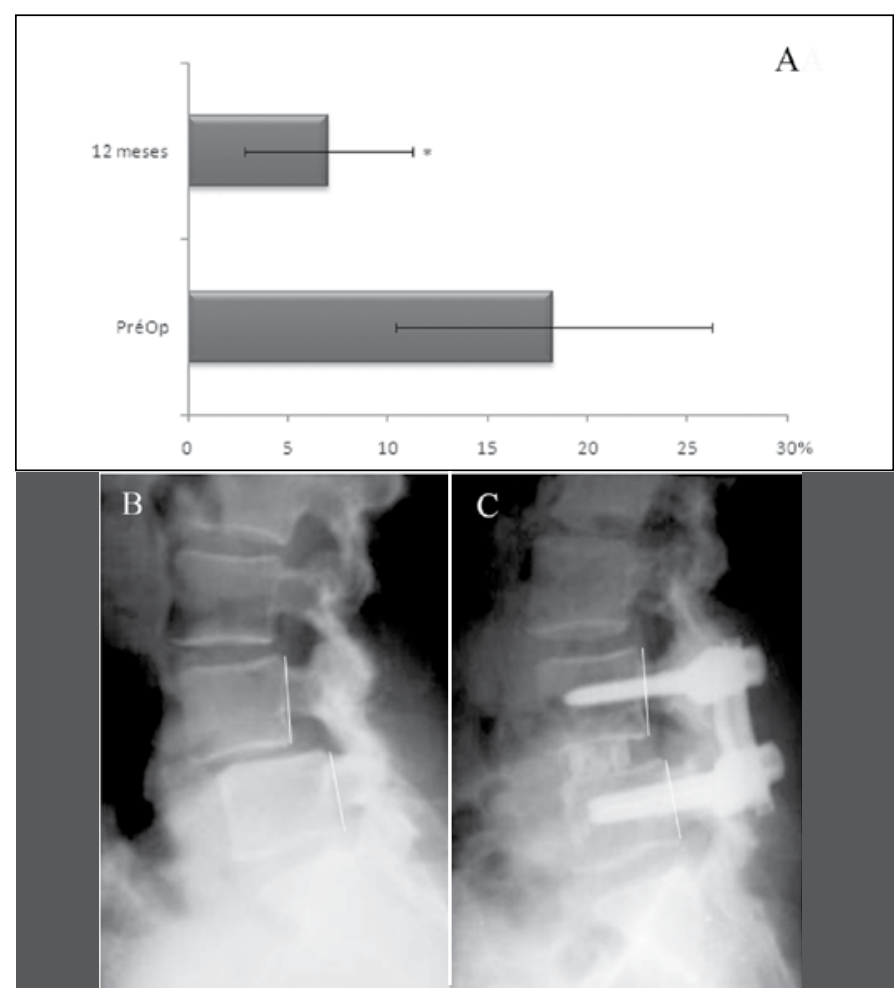

Figura 3. Redução da espondilolistese após o procedimento. (A) Valores relativos médios de escorregamento da vértebra no nível afetado em exames pré-operatórios e de 12 meses após cirurgia. * $p<0,001$. (B) Imagem de raios-x pré-operatório e (C) de 12 meses mostrando exemplo de caso com redução da espondilolistese com fusão intersomática e suplementação posterior. 

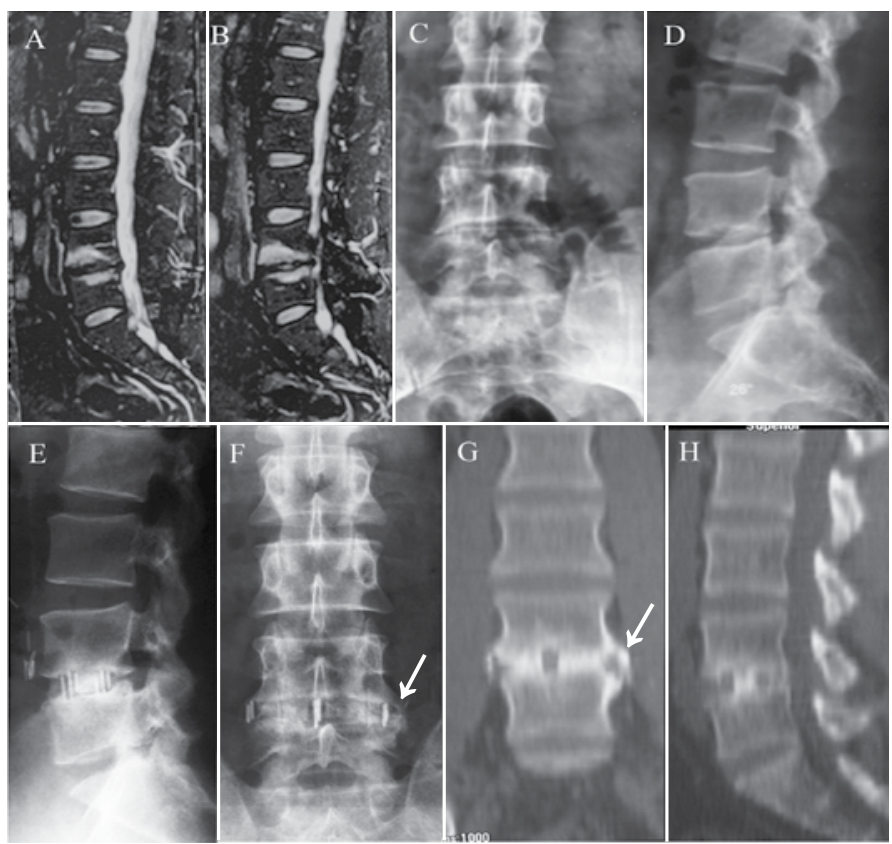

Figura 4. Exemplo de caso evidenciando fusão intersomática. (A) e (B) Imagens de ressonância magnética pré-operatória. (C) Imagem ântero-posterior e (D) lateral de exame de raios-x pré-operatório. (E) Imagem lateral e (F) ântero-posterior de exame exame de raios-x pré-operatorio. (E) Imagem lateral e $(F)$ ântero-posterior de exame
de raios-x após de 12 meses de cirurgia. $(G)$ Reconstrução coronal e $(H)$ sagital de tomografia computadorizada após de 12 meses de cirurgia. Setas evidenciam o sinal sentinela de crescimento ósseo por fora do implante.

Tabela 2. Cirurgias de revisão.

\begin{tabular}{c|c}
\hline Causa & Casos \\
\hline Estenose persistente & $3(6,5 \%)$ \\
\hline Afundamento do implante & $3(6,5 \%)$ \\
\hline Falha de suplementação & $1(2,8 \%)$ \\
\hline Total & $7(15.2 \%)$ \\
\hline
\end{tabular}

\section{DISCUSSÃO}

A abordagem lateral à coluna tóraco-lombar anterior tem mostrado resultados clínicos e radiológicos satisfatórios, aliando-se ainda as vantagens de uma cirurgia minimamente invasiva. No caráter intra-operatório, não há necessidade de um cirurgião geral, pois não se manipula o peritônio e os grandes vasos; a dissecção ocorre sem prejuízo da percepção de profundidade por acontecer sob visão tridimensional; é evitada a lesão do plexo de nervo hipogástrico superior e a assim, a ocorrência de ejaculação retrógrada. Além, os relatos mostram baixa perda sanguínea, reduzida taxa de complicações e rápido retorno às atividades diárias do paciente $7,8,30,31$.

0 acesso lateral consiste em uma técnica relativamente nova que aparece como uma opção minimamente invasiva e biomecanicamente atrativa, na qual o acesso direto ao disco intervertebral é conseguido lateralmente através de retratores tubulares progressivos, através de abordagem retroperitoneal através das fibras do músculo psoas maior ${ }^{5}$. Como item de segurança indispensável, a monitoração eletromiográfica em tempo real (EMG) é realizada para orientar a passagem segura através do psoas ${ }^{32}$, sítio de passagem do plexo lombar.

Ao contrário das abordagens anteriores diretas ou posteriores, o acesso lateral mantém os ligamentos longitudinais anterior e posterior intactos, e permite a colocação de um implante de grande área de contato com o platô vertebral, resultando em uma construção biomecanicamente bem estável ${ }^{33,34}$. A técnica cirúrgica adequada consiste na ampla remoção do disco e liberação do anel contralateral ao acesso ${ }^{5}$. Este processo não só garante uma enxertia apropriada, mas também permite a distração uniforme do espaço discal, que restaura a altura do disco e facilita a redução da deformidade por meio da ligamentotaxia $a^{5,7,8,35}$.
Do ponto de vista biomecânico pode-se lembrar de que $80 \%$ da compressão, torção, e as forças de cisalhamento são transmitidas através da coluna anterior ${ }^{11,12}$. Com uma grande área para o crescimento ósseo, o espaçador e o enxerto são colocados à frente do eixo instantâneo de rotação e fica exposto à compressão, ao invés vez de forças de tração, criando um ambiente favorável para a estabilização e consolidação óssea ${ }^{34,36}$. Assim, as taxas de fusão após fusão intersomática são consideravelmente mais elevadas do que aquelas observados após fusão posterolateral ${ }^{37-41}$, sendo que as taxas de pseudartrose após fusão posterolateral variam em torno de $20 \%{ }^{42,43}$. A taxa de fusão da nossa série é semelhante às encontrados em outros estudos, variando de 90 a 100\%5,10,35,44,45.

As técnicas de fusão intersomática, assim como o acesso lateral, tem como objetivo preservar a capacidade para suporte de carga da coluna, restaurar o alinhamento coronal e sagital ${ }^{46-49}$; restaurar a altura do disco, espaço e dimensões foraminais ${ }^{7,35}$; e usar a compressão e a estabilidade para aumentar a probabilidade de fusão ${ }^{34,36}$

Assim como mostrado através dos exemplos mostrados e resultados de alívio de dor neurogênica, o procedimento prevê a descompressão indireta necessária para o tratamento da estenose central e/ou lateral (Figura 5), evitando a necessidade de ressecção direta de elementos posteriores, e então, as morbidades associadas. Porém, a descompressão indireta poderá ser limitada em casos de estenose congênita e/ou anquilose facetária ${ }^{7}$. Seu efeito pode também ser reduzido pelo afundamento do implante ${ }^{7,50}$, que quando presente acontece no período pós-operatório mais frequentemente em pacientes com perda óssea devido à osteoporose ${ }^{31}$, e como mostrado aqui, pode levar à cirurgia de revisão para estabilização do nível e descompressão direta das estruturas nervosas.

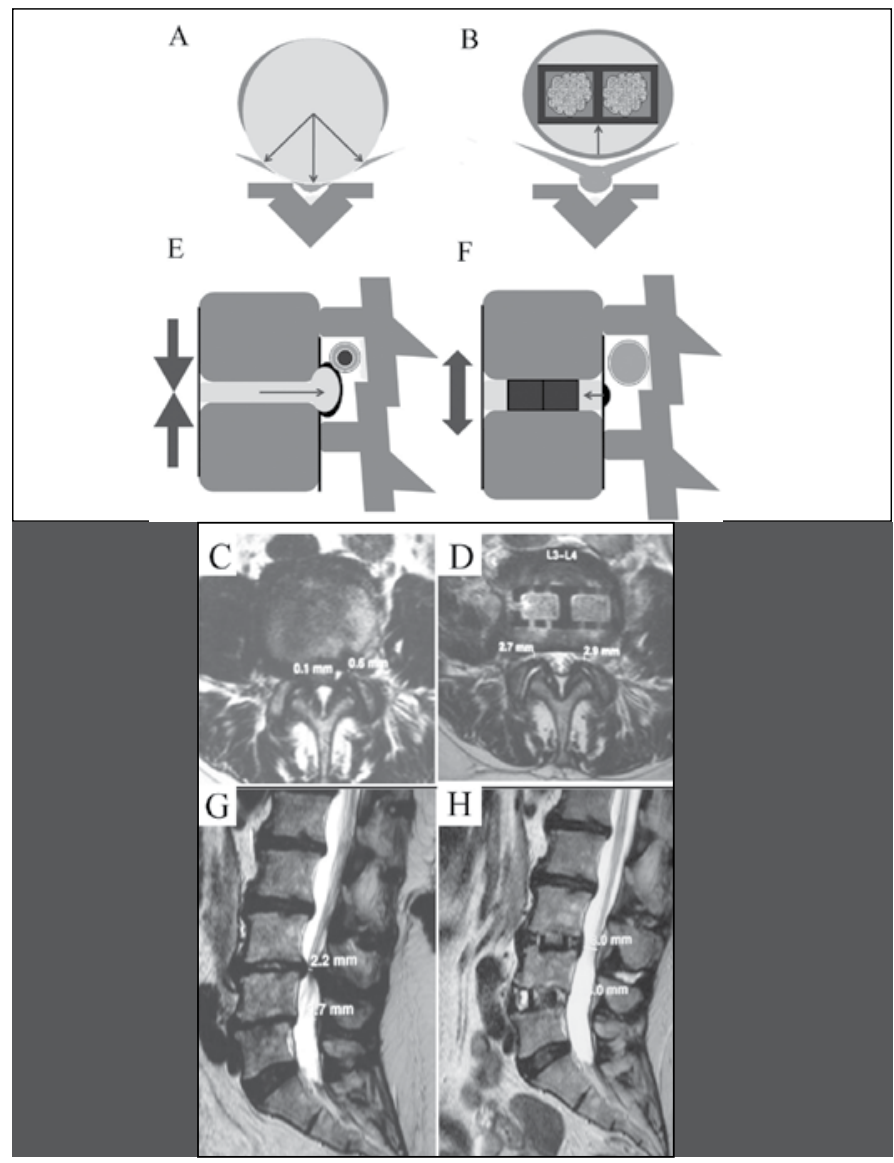

Figura 5 - Conceito e caso de descompressão indireta em visão sagital e axial. (A) e (C) Visão axial da diminuição do espaço discal com estenose central e foraminal. (B) e (D) Visão axial do implante intersomático com restauração do espaço discal e ligamentotaxia provocando a descompressão indireta. (E) e (G) Visão sagital da e ligamentotaxia provocando a descompressão indireta. (E) e $(G)$ Visão sagital da
diminuição do espaço discal com estenose central e foraminal. (F) e (H) Visão sagital do implante intersomático com restauração do espaço discal e ligamentotaxia provocando a descompressão indireta. 
Além da descompressão, o alongamento da coluna anterior promove correção nos planos coronal, sagital e axial da coluna lombar com múltiplas condições degenerativa, como na escoliose degenerativa ${ }^{31,44,51,52}$ (Figura 6).
A utilização da fusão intersomática por via lateral minimamente invasiva se mostrou um procedimento seguro e eficaz para alcançar a artrodese tóraco-lombar e promover a descompressão dos elementos neurais.

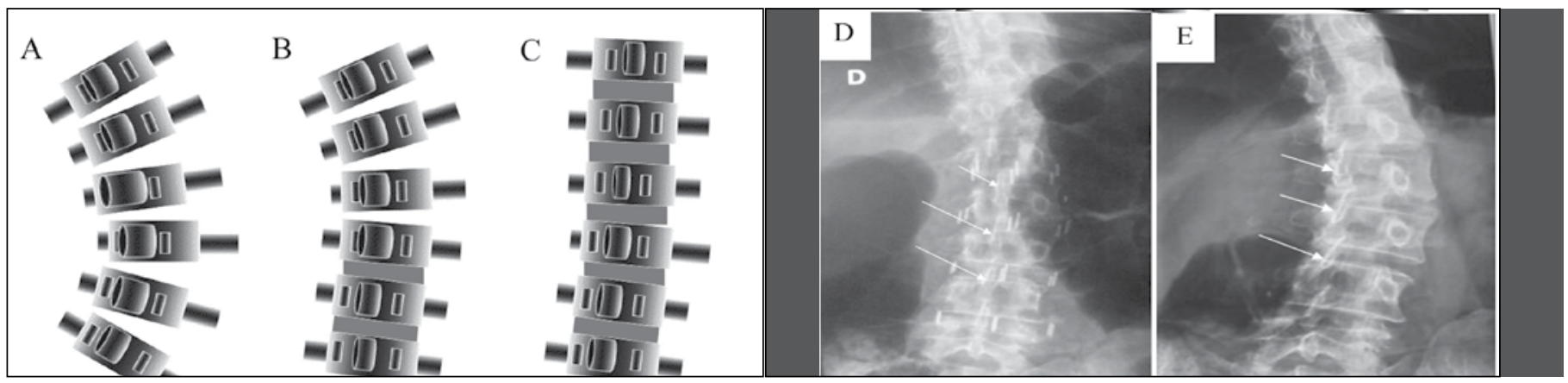

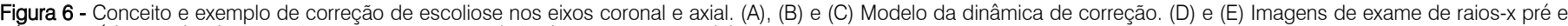
pós-operatórias revelando correção, setas mostram posição dos processos espinhosos.

\section{REFERÊNCIAS}

1. Tay BB, Berven S. Indications, techniques, and complications of lumbar interbody fusion. Semin Neurol. 2002;22(2):221-30.

2. Cloward RB. Posterior lumbar interbody fusion updated. Clin Orthop Relat Res. 1985; (193):16-9.

3. Harms J, Jeszenszky D. The unilateral, transforaminal approachfor posterior lumbar interbody fusion. Orthop Traumatol. 1998;6(1):88-99.

4. Obenchain TG. Laparoscopic lumbar discectomy: case report. J Laparoendosc Surg. 1991;1(3):145-9

5. Ozgur BM, Aryan HE, Pimenta L, Taylor WR. Extreme Lateral Interbody Fusion (XLIF): a novel surgical technique for anterior lumbar interbody fusion. Spine J. 2006:6(4):435-43.

6. McAfee PC Phillips FM, Andersson G Buvenenadran A Kim CW Lauryssen C et al. Minimally invasive spine surgery. Spine (Phila Pa 1976). 2010 Dec 15;35(26 Suppl):S271-3.

7. Oliveira L, Marchi L, Coutinho E, Pimenta L. A radiographic assessment of the ability of the extreme lateral interbody fusion procedure to indirectly decompress the neural elements. Spine (Phila Pa 1976). 2010;35(26 Suppl):S331-7.

8. Billinghurst J, Akbarnia BA. Extreme lateral interbody fusion - XLIF. Curr Orthop Pract. 2009;20:238-51.

9. Oliveira L, Marchi L, Coutinho E, Abdala N, Pimenta L. The use of rh-BMP2 in Standalone eXtreme lateral interbody fusion (XLIF®): clinical and radiological results after 24 months follow-up. WSCJ. 2010;1(1):19-25

10. Ozgur BM, Agarwal V, Nail E, Pimenta L. Two-year clinical and radiographic success of minimally invasive lateral transpsoas approach for the treatment of degenerative lumbar conditions. SAS J. 2010;1(4):41-6

11. Harms, J. Screw-Threaded Rod System in Spinal Fusion Surgery. In: Dorman TA. Spine: State of the Art Reviews. Hanley \& Belfus; 1992, pp. 541-575.

12. Yang SW, Langrana NA, Lee CK. Biomechanics of lumbosacral spinal fusion in combined compression-torsion loads. Spine (Phila Pa 1976). 1986;11(9):937-41.

13. Brantigan JW, Steffee AD. A carbon fiber implant to aid interbody lumbar fusion. Two-year clinical results in the first 26 patients. Spine (Phila PA 1976). 1993;18(14):2106-7.

14. Gejo R, Matsui $H$, Kawaguchi $Y$, Ishihara $H$, Tsuji $H$. Serial changes in trunk muscle performance after posterior lumbar surgery. Spine (Phila Pa 1976). 1999:24(10):1023-8.

15. Kawaguchi Y, Yabuki S, Styf J, Olmarker K, Rydevik B, Matsui H, Tsuji H. Back muscle injury after posterior lumbar spine surgery. Topographic evaluation of intramuscular pressure and blood flow in the porcine back muscle during surgery. Spine (Phila Pa 1976). 1996;21(22):2683-8

16. Rantanen J, Hurme M, Falck B, Alaranta H, Nykvist F, Lehto M, Einola S, Kalimo H. The lumbar multifidus muscle five years after surgery for a lumbar intervertebral disc herniation. Spine (Phila Pa 1976). 1993;18(5):568-74.

17. Sihvonen T, Herno A, Paliärvi L, Airaksinen O, Partanen J, Tapaninaho A. Local denervation atrophy of paraspinal muscles in postoperative failed back syndrome. Spine (Phila Pa 1976). 1993:18(5):575-81.

18. Crock HV. Anterior lumbar interbody fusion: indications for its use and notes on surgical technique. Clin Orthop Relat Res. 1982;(165):157-63.

19. Lund T, Oxland TR, Jost B, Cripton P, Grassmann S, Etter C, Nolte LP. Interbody cage stabilisation in the lumbar spine: biomechanical evaluation of cage design, posterio instrumentation and bone density. J Bone Joint Surg Br. 1998;80(2):351-9.

20. Resnick DK, ChoudhriTF, Dailey AT, Groff MW, Khoo L, Matz PG, et al. Guidelines for the performance of fusion procedures for degenerative disease of the lumbar spine. Part 11 interbody techniques for lumbar fusion. J Neurosurg Spine. 2005;2(6):692-9.

21. Madan SS, Harley JM, Boeree NR. Anterior lumbar interbody fusion: does stable anterio fixation matter? Eur Spine J. 2003;12(4):386-92

22. Oliveira $L$, Marchi $L$, Coutinho E, Pimenta $L$. Standalone anterior interbody fusion procedure for the treatment of low-grade spondylolisthesis: a case series. WSC J. 2010;1(3):194-200.

23. Sasso RC, Kenneth Burkus J, LeHuec JC. Retrograde ejaculation after anterior lumbar interbody fusion: transperitoneal versus retroperitoneal exposure. Spine (Phila Pa 1976). 2003;28(10):1023-6.

24. Tiusanen H, Seitsalo S, Osterman K, Soini J. Anterior interbody lumbar fusion in severe low back pain. Clin Orthop Relat Res. 1996;(324):153-63.

25. Baker JK, Reardon PR, Reardon MJ, Heggeness MH. Vascular injury in anterior lumbar surgery. Spine (Phila Pa 1976). 1993;18(15):2227-30.

26. Farkas JC Chapuis C Combe S, Silsiguen M, Marzelle J, Laurian C, et al. A randomised controlled trial of a low-molecular-weight heparin (Enoxaparin) to prevent deep-vein thrombosis in patients undergoing vascular surgery. Eur J Vasc Surg. 1993;7(5):554-60.

27. Nguyen HV, Akbarnia BA, van Dam BE, Raiszadeh K, Bagheri R, Canale S, et al. Anterior exposure of the spine for removal of lumbar interbody devices and implants. Spine (Phila Pa 1976). 2006;31(21):2449-53.
28. Pimenta L, Díaz RC, Guerrero LG. Charité lumbar artificial disc retrieval: use of a lateral minimally invasive technique. Technical note. J Neurosurg Spine. 2006;5(6):556-61.

29. Vigatto R, Alexandre NM, Correa Filho HR. Development of a Brazilian Portuguese version of the Oswestry Disability Index: cross-cultural adaptation, reliability, and validity. Spine (Phila Pa 1976). 2007:32(4):481-6.

30. Rodgers WB, Gerber EJ, Patterson J. Intraoperative and early postoperative complications in extreme lateral interbody fusion: an analysis of 600 cases. Spine (Phila Pa 1976). 2011;36(1):26-32.

31. Tormenti MJ, Maserati MB, Bonfield CM, Okonkwo DO, Kanter AS. Complications and radiographic correction in adult scoliosis following combined transpsoas extreme lateral interbody fusion and posterior pedicle screw instrumentation. Neurosurg Focus. 2010:28(3):E7.

32. Uribe JS, Arredondo N, Dakwar E, Vale FL. Defining the safe working zones using the minimally invasive lateral retroperitoneal transpsoas approach: an anatomical study. J Neurosurg Spine. 2010 Aug;13(2):260-6. Erratum in: J Neurosurg Spine. 2010;13(6):798.

33. Grant JP, Oxland TR, Dvorak MF. Mapping the structural properties of the lumbosacra vertebral endplates. Spine (Phila Pa 1976). 2001;26(8):889-96.

34. Voor MJ, Mehta S, Wang M, Zhang YM, Mahan J, Johnson JR. Biomechanical evaluation of posterior and anterior lumbar interbody fusion techniques. J Spinal Disord. 1998:11(4):328-34

35. Youssef JA, McAfee PC, Patty CA, Raley E, DeBauche S, Shucosky E, Chotikul L. Minimally invasive surgery: lateral approach interbody fusion: results and review. Spine (Phila Pa 1976). 2010;35(26 Suppl):S302-11.

36. Evans JH. Biomechanics of lumbar fusion. Clin Orthop Relat Res. 1985;(193):38-46.

37. Ma GW. Posterior lumbar interbody fusion with specialized instruments. Clin Orthop Relat Res. 1985:(193):57-63.

38. Steffee AD, Sitkowski DJ. Posterior lumbar interbody fusion and plates. Clin Orthop Relat Res. 1988;227:99-102.

39. Fujimaki A, Crock HV, Bedbrook GM. The results of 150 anterior lumbar interbody fusion operations performed by two surgeons in Australia. Clin Orthop Relat Res. 1982;(165):164-7.

40. Blumenthal SL, Baker J, Dossett A, Selby DK. The role of anterior lumbar fusion for internal disc disruption. Spine (Phila Pa 1976). 1988:13(5):566-9.

41. Benton BF, Calandruccio RA. Surgical technic of anterior lumbar fusion. Am Surg. 1966;32(2):134-6.

42. Parker LM, Murrell SE, Boden SD, Horton WC. The outcome of posterolateral fusion in highly selected patients with discogenic low back pain. Spine (Phila PA 1976). 1996;21(16):1909-16.

43. Turner JA, Ersek M, Herron L, Haselkorn J, Kent D, Ciol MA, Deyo R. Patient outcomes after lumbar spinal fusions. JAMA. 1992:268(7):907-11.

44. Rodgers WB, Cox $C$, Gerber E. Experience and early results with a minimally invasive technique for anterior column support through eXtreme lateral interbody fusion (XLIF®). US Musculoskelet Dis. 2007;2(1):28-32.

45. Rodgers WB, Gerber EJ, Patterson JR. Fusion after minimally disruptive anterior lumbar interbody fusion: Analysis of extreme lateral interbody fusion by computed tomography SAS J. 2010;4:63-6.

46. Karikari 1O, Nimjee SM, Hardin CA, Hughes BD, Hodges TR, Mehta Al, et al. Extreme lateral interbody fusion approach for isolated thoracic and thoracolumbar spine diseases: initial clinical experience and early outcomes. J Spinal Disord Tech. 2011:24(6):368-75.

47. Dakwar E, Cardona RF, Smith DA, Uribe JS. Early outcomes and safety of the minimally invasive, lateral retroperitoneal transpsoas approach for adult degenerative scoliosis. Neurosurg Focus. 2010;28(3):E8.

48. Isaacs RE, Hyde J, Goodrich JA, Rodgers WB, Phillips FM. A prospective, nonrandomized, multicenter evaluation of extreme lateral interbody fusion for the treatment of adult degenerative scoliosis: perioperative outcomes and complications. Spine (Phila Pa 1976). 2010:35(26 Suppl):S322-30.

49. Sharma AK, Kepler CK, Girardi FP, Cammisa FP, Huang RC, Sama AA. Lateral lumbar interbody fusion: clinical and radiographic outcomes at 1 year: a preliminary report. $J$ Spinal Disord Tech. 2011;24(4):242-50

50. Karikari IO, Grossi PM, Nimjee SM, Hardin C, Hodges TR, Hughes BD, et al. Minimally invasive lumbar interbody fusion in patients older than 70 years of age: analysis of periand postoperative complications. Neurosurgery 2011:68(4):897-902.

51. Benglis DM, Elhammady MS, Levi AD, Vanni S. Minimally invasive anterolateral approaches for the treatment of back pain and adult degenerative deformity. Neurosurgery. 2008;63(3 Suppl):191-6.

52. Mundis GM, Akbarnia BA, Phillips FM. Adult deformity correction through minimally invasive lateral approach techniques. Spine (Phila Pa 1976). 2010;35(26 Suppl):S312-21. 